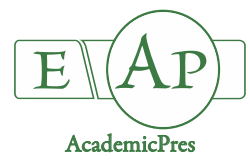

\title{
Genetic Diversity and Population Structure Analysis of Sand Pear (Pyrus pyrifolia) 'Nakai' Varieties Using SSR and AFLP Markers
}

\author{
Jie $\mathrm{CAO}^{1 \mathrm{a}}$, Zhongcheng ZHOU ${ }^{2 \mathrm{~b}}$, Junfan $\mathrm{TU}^{3 \mathrm{c}}$, Shuiyuan $\mathrm{CHENG}^{4}$, \\ Jinglei $\mathrm{YAO}^{1}$, Feng $\mathrm{XU}^{1 *}$, Guiyuan $\mathrm{WANG}^{1}$, Jian $\mathrm{ZHANG}^{1}$, Jiabao $\mathrm{YE}^{1}$, \\ Yongling $\mathrm{LIAO}^{1}$, Weiwei $\mathrm{ZHANG}^{1}$, Zexiong $\mathrm{CHEN}^{5 * *}$ \\ 'Yangtze University, College of Horticulture and Gardening, Jingzhou 434025, Hubei, China; caojie1302@163.com; Brest005@163.com; \\ xufeng198@126.com (*corresponding author);guiyuanwang@163.com; zhangjian840000@163.com; yejiabao13@163.com; \\ liaoyongling@yeah.net;wwzhangchn@163.com \\ ${ }^{2}$ Hubei Ecology Polytechnic College, Department of Forestry Ecology, Wuhan 430070, China; 1806725680@qq.com \\ ${ }^{3}$ Hubei Academy of Agricultural Sciences, Fruit and Tea Research Institute, Wuban 430064, China; tujunfan2005@126.com \\ ${ }^{4}$ Wuban Polytechnic University, National R\&D Center for Se-rich Agricultural Products Processing Technology, \\ Wuban 430023, China; s_y_cheng@sina.com \\ ${ }^{5}$ Chongqing University of Arts and Sciences, Research Institute for Special Plants, Chongqing 402160, \\ China; chenzexiong1979@163.com (**correspondingauthor) \\ a,bc, These authors contributed equally to this study
}

\begin{abstract}
In this study, the technologies of simple sequence repeat (SSR) and amplified fragment length polymorphism (AFLP) markers were used to analyze the genetic diversity of 30 sand pear (Pyrus pyrifolia) 'Nakai' varieties. Ten pairs of SSR polymorphic primers were selected to amplify $P$. pyrifolia 'Nakai' varieties. A total of 90 alleles were detected. The polymorphism information content index was between 0.5578 and 0.8423 , with an average of 0.7585 . The selected 10 pairs of AFLP primer combinations were used to amplify the analyzed pear varieties, and 1,046 polymorphic loci were detected. The average amplification results of each primer combination detected 105 bands with an average polymorphism percentage of 86.46\%. The combined data of SSR and AFLP analysis showed that the analyzed P. pyrifolia 'Nakai' varieties were characterized by extremely rich genetic diversity and were highly representative. According to the results of SSR, AFLP, and SSR+AFLP cluster analysis, the analyzed $P$. pyrifolia 'Nakai' varieties can be categorized into three clusters. The results of genetic structure showed that the hybridization between these $P$. pyrifolia 'Nakai' varieties resulted in the heterozygosity of genotypes. In addition, we found that 'Nijisseik', 'Ejima', and 'Fuli' are good parent resources among the pear varieties through observing the genetic background of the analyzed pear varieties. This study reveals the genetic diversity levels of $P$. pyrifolia 'Nakai' varieties at the molecular level, which was important in molecular identification and protection of pear germplasm resources, as well as pear variety breeding and genetic improvement.
\end{abstract}

Keywords: cluster analysis; genetic polymorphism; genetic structure; molecular markers; pear varieties

\section{Introduction}

Pear belongs to the Rosaceae family and is mainly divided into white pear, sand pear, autumn pear, and other varieties. Most pear varieties originating in East Asia can be divided into five groups, namely, Ussurian pear, Chinese white pear, Chinese sand pear, Xinjiang pear, and Japanese pear, according to their geographical distribution (Teng and
Tanabe, 2004). As the origin of the pear, China is the largest pear-producing country in the world and one of the main origin centers of pear plants. The pear variety resources are abundant in China with complex and changeable phenotypes (Zhang et al., 2016). The breeding and popularization of pear varieties accelerate the exchange of germplasm resources in different places and also increase the genetic diversity of pear varieties. However, with the increase of pear varieties, it is more and more difficult to 
distinguish different varieties, which are only depending on phenotypic characterization and protein marker analysis. Therefore, identifying pear varieties and determining genetic correlation are necessary in cultivating new varieties and in collecting and preserving germplasm resources (Tana et al., 2015; Yue et al., 2018).

The traditional morphological feature identification method is difficult to apply and tends to change due to environmental factors. This method, which requires strong professional basic knowledge, is also time consuming and laborious (Jinbo et al., 2011). However, DNA molecular marker technology can be used without the influence of environmental conditions. This technology can quickly and accurately identify the genetic specificity of varieties at the molecular level, and overcome the deficiencies of traditional morphological markers, such as a long identical cycle, large amount of errors and requiring special skills acquired through extensive experience (Poczai et al., 2013). Therefore, this technology has been widely applied in the analysis of genetic diversity, variety identification, and establishment of fingerprints in various fruit trees such as strawberries (Sánchez-Sevilla et al., 2015), hawthorn (Güney et al., 2018), citrus (Rao et al., 2008), and peach (Cheng and Huang, 2009). At present, various types of molecular markers have been widely applied in the study of genetic diversity of pear varieties. In pear tree fingerprinting studies, random amplification of polymorphic DNA (RAPD) (Lisek and Rozpara, 2010), amplified fragment length polymorphism (AFLP) (Yamamoto et al., 2007), simple sequence repeat (SSR) (Yue et al., 2014; Puskás et al., 2016), inter-simple sequence repeat amplification (ISSR) (Monte-Corvo et al., 2001), and sequence-related amplification polymorphism (SRAP) (Zhang et al., 2012) were all used to evaluate genetic variability and identify germplasm resources. Therefore, in this study, SSR and AFLP markers were used to analyze the genetic diversity and population structure of $P$. pyrifolia 'Nakai' varieties to reveal their genetic diversity at the molecular level and provide a basis for molecular identification of pear variety resources and protection of variety rights, as well as pear variety breeding and genetic improvement.

\section{Materials and Methods}

\section{Materials}

A total of 30 P. pyrifolia 'Nakai' varieties were collected, which included 23 Chinese cultivars, 5 Japanese cultivars and 2 Korean cultivars (Table 1). These were provided by Yangtze University and Fruit and Tea Research Institute of Hubei Academy of Agricultural Sciences.

DNA extraction and molecular marker polymorphism screening

The leaves were frozen in liquid nitrogen, and the DNA was extracted using CATB method (Ye et al., 2017). The quality and concentration of DNA were determined using $1 \%$ agarose gels and a NanoPhotometer ${ }^{\circ}$ spectrophotometer (Implen, CA, USA), respectively. The extracted DNAs were diluted to $40 \mathrm{ng} \mu \mathrm{L}^{-1}$ for PCR reaction.
To fully reflect the genetic diversity of $P$. pyrifolia 'Nakai' varieties, the SSR primers refer to the genetic linkage map of pears constructed by Terakami et al. (2009) and Celton et al. (2009). Seventeen pairs of SSR primers distributed in 17 linkage groups of pears were selected for screening, and the primer sequences were reported by Yamamoto et al. (2002) and Liebhard et al. (2002) (Table 2). Primer pairs were prescreened on four genotypes ('Eli 2', 'Housui', 'Jingli 1', and 'Wonhwang') in order to identify primers that generated clear bands for further amplification reactions for $P$. pyrifolia 'Nakai' varieties.

Finally, 10 pairs of SSR primers and 10 pairs of appropriately combined AFLP primers (Table 3) were determined for further amplification of 30 sand pear DNA samples. All primers were synthesized by Sangon Biological Engineering Technology and Service Co. Ltd, Shanghai.

\section{SSR analysis}

PCR reactions were carried out in a $20 \mu \mathrm{L}$ mixture: 14.8 $\mathrm{uL}$ of $\mathrm{ddH}_{2} \mathrm{O}, 0.4 \mathrm{uL}$ of $\mathrm{dNTP}$ mixture, $2 \mathrm{uL}$ of $10 \times \mathrm{PCR}$ Buffer, $0.3 \mathrm{uL}$ of forward primer $(20 \mathrm{uM}), 0.3 \mathrm{uL}$ of reverse primer $(20 \mathrm{uM}), 2 \mu \mathrm{L}$ of DNA template and $0.2 \mathrm{uL}$ of Taq DNA polymerase (TaKaRa, Dalian, China). The SSR PCR reaction was carried out as follows: $94^{\circ} \mathrm{C}$ for $5 \mathrm{~min}$ for the initial denaturing step, followed by 35 cycles at $94^{\circ} \mathrm{C}$ for 30 $\mathrm{s}, 55^{\circ} \mathrm{C}$ for $35 \mathrm{~s}$, and $72{ }^{\circ} \mathrm{C}$ for $40 \mathrm{~s}$ for denaturing, annealing, and extension, respectively, followed by a final extension step at $72^{\circ} \mathrm{C}$ for $3 \mathrm{~min}$.

\section{AFLP analysis}

Frequent cutter $M s e \mathrm{I}$ and rare cutter EcoR I endonucleases source of purchase were used for restriction digestion of genomic DNA, restriction and ligation were then carried out together. Restriction and ligation mixture were carried out in a total volume of $20 \mu \mathrm{L}: 2 \mu \mathrm{L}$ of $10 \times$ AFLP digest-ligation buffer, $1.8 \mu \mathrm{L}$ of AFLP digestligation enzyme mix, $1 \mu \mathrm{L}$ of $E c o \mathrm{R}$ I adaptor $(10 \mu \mathrm{M}), 1 \mu \mathrm{L}$ of $\mathrm{Mse}$ I adaptor $(10 \mu \mathrm{M}), 4 \mu \mathrm{L}$ of DNA template and 10.2 $\mu \mathrm{L}$ of $\mathrm{ddH}_{2} \mathrm{O} ; 25^{\circ} \mathrm{C} 5 \mathrm{~h}$.

The total amount of pre-amplification was $20 \mu \mathrm{L}: 10 \mu \mathrm{L}$ of $2 \times$ PCR Mix, $1 \mu \mathrm{L}$ of E00 $(20 \mu \mathrm{M}), 1 \mu \mathrm{L}$ M00 $(20 \mu \mathrm{M}), 4$ $\mu \mathrm{L}$ of restriction-ligation products and $4 \mu \mathrm{L}$ of $\mathrm{ddH}_{2} \mathrm{O}$. The DNA amplification was performed using the following program: $94^{\circ} \mathrm{C}$ for $3 \mathrm{~min}$ for the initial denaturing step, followed by 30 cycles at $94^{\circ} \mathrm{C}$ for $30 \mathrm{~s}, 50{ }^{\circ} \mathrm{C}$ for $35 \mathrm{~s}$, and $72{ }^{\circ} \mathrm{C}$ for $1 \mathrm{~min}$ for denaturing, annealing, and extension, respectively. The quality and concentration of preamplification PCR products were determined using $1 \%$ agarose gels and a NanoPhotometer ${ }^{\circledR}$ spectrophotometer (Implen, CA, USA).

Selective amplification were carried out in a $20 \mu \mathrm{L}$ mixture: $10 \mu \mathrm{L}$ of $2 \times \mathrm{PCR}$ Mix, $1 \mu \mathrm{L}$ of E primer $(20 \mu \mathrm{M}), 1$ $\mu \mathrm{L}$ of $\mathrm{M}$ primer $(20 \mu \mathrm{M}), 5 \mu \mathrm{L}$ Pre-PCR products (Pre-PCR products were diluted 20-fold and used as DNA template for selective amplification) and $3 \mu \mathrm{L} \mathrm{ddH}_{2} \mathrm{O}$. Selective amplification was carried out using the following touchdown program: $95^{\circ} \mathrm{C}$ for $5 \mathrm{~min}, 95^{\circ} \mathrm{C}$ for $35 \mathrm{~s}, 60^{\circ} \mathrm{C}$ for $35 \mathrm{~s}, 72^{\circ} \mathrm{C}$ for $1 \mathrm{~min}$, followed by 12 cycles of each with $0.7{ }^{\circ} \mathrm{C}$ lowering of annealing temperature and finally 23 cycles of $94^{\circ} \mathrm{C}$ for $30 \mathrm{~s}, 56^{\circ} \mathrm{C}$ for $30 \mathrm{~s}$ and $72^{\circ} \mathrm{C}$ for $1 \mathrm{~min}$. 
972

Table 1. Names, sources and parentage of $P$. pyrifolia 'Nakai' varieties

\begin{tabular}{|c|c|c|c|}
\hline Code & Name of varieties & Sources & Percentage \\
\hline $\mathrm{Z} 01$ & Chuxiang & China & Laiyangcili $\times$ Ejima \\
\hline Z02 & Cuiguan & China & Kousui $\times$ (hangqing $\times$ Shinseiki) \\
\hline $\mathrm{Z} 03$ & Deshengxiang & China & Landrace \\
\hline Z04 & Eli 1 & China & Jinshuisu $\times$ Fuli \\
\hline $\mathrm{Z} 05$ & Eli 2 & China & Zhongxiang $\times($ Fuli $\times$ Beurre Giffard $)$ \\
\hline Z06 & Ninomiyahuri & Japan & Yali $\times$ Zhenyu \\
\hline $\mathrm{Z} 07$ & Housui & Japan & Bayun $\times$ Cuixing \\
\hline Z08 & Huali 1 & China & Syounan $\times$ Ejima \\
\hline Z09 & Huangguan & China & Sheinseiki×Xuehuali \\
\hline $\mathrm{Z} 10$ & Whangkeumbae & Korea & Nijisseik $\times$ Niitaka \\
\hline $\mathrm{Z} 11$ & Jinshui 2 & China & Ejima $\times$ Chojuro \\
\hline $\mathrm{Z} 12$ & Jinshui 3 & China & Syouna $\times$ Ejima \\
\hline $\mathrm{Z} 13$ & Jinshuiqiu & China & Okusankichi×Laiyangcili \\
\hline $\mathrm{Z} 14$ & Jinshuisu & China & Jinshui $1 \times$ Xinglongmali \\
\hline $\mathrm{Z} 15$ & Jingli 1 & China & Landrace \\
\hline Z16 & Laiyangcili & China & Landrace \\
\hline $\mathrm{Z} 17$ & Lvbaoshi & China & Sheinseiki $\times$ Zaosu \\
\hline $\mathrm{Z} 18$ & Qingxiang & China & Sanhua $\times$ Sheinseiki \\
\hline Z19 & Qiuyue & Japan & Kousui $\times($ Niitaka $\times$ Kousui $)$ \\
\hline $\mathrm{Z} 20$ & Wuzili & China & Yali $\times$ Rushanbali \\
\hline $\mathrm{Z} 21$ & Xizilv & China & (Bayun $\times$ Hangqing $) \times$ Sheinseiki \\
\hline $\mathrm{Z} 22$ & Niitaka & Japan & Imanuraaki×Amanokawa \\
\hline $\mathrm{Z} 23$ & Xueqiu & China & Landrace \\
\hline $\mathrm{Z} 24$ & Yulv & China & Taibai $\times$ Laiyangcili \\
\hline $\mathrm{Z} 25$ & Yuxiang & China & Jinshuisu $\times$ Fuli \\
\hline $\mathrm{Z} 26$ & Wonhwang & Korea & Waseaka×Okusankichi \\
\hline $\mathrm{Z} 27$ & Zaomeisu & China & Zaosu $\times$ Shinseiki \\
\hline $\mathrm{Z} 28$ & Zaosu & China & qianliang $\times$ Pingguoli \\
\hline $\mathrm{Z} 29$ & Chojuro & Japan & Landrace \\
\hline $\mathrm{Z} 30$ & Zhongxiang & China & Landrace \\
\hline
\end{tabular}

Table 2. Sequences of 17 pairs of primers used in SSR marker

\begin{tabular}{|c|c|c|c|c|}
\hline Code & Locus name & Primer pairs sequences $\left(5^{\prime}-3^{\prime}\right)$ & Locus name & Primer pairs sequences $\left(5^{\prime}-3^{\prime}\right)$ \\
\hline $\mathrm{Pl}$ & NH013a-F & GGTTTGAAGAGGAATGAGGAG & NH013a-R & CATTGACTTTAGGGCACATTTC \\
\hline P2 & BGT23b-F & CACATTCAAAGATTAAGAT & BGT23b-R & ACTCAGCCTTTTTTTCCCAC \\
\hline P3 & $\mathrm{CH} 03 \mathrm{gl} 2-\mathrm{F}$ & GCGCTGAAAAAGGTCAGTTT & $\mathrm{CH} 03 \mathrm{gl} 12-\mathrm{R}$ & CAAGGATGCGCATGTATTTG \\
\hline P4 & NH01la-F & GGTTCACATAGAGAGAGAGAG & NH011a-R & TTTGCCGTTGGACCGAGC \\
\hline P5 & $\mathrm{CH} 04 \mathrm{~g} 09-\mathrm{F}$ & TTGTCGCACAAGCCAGTTTA & $\mathrm{CH} 04 \mathrm{~g} 09-\mathrm{R}$ & GAAGACTCATGGGTGCCATT \\
\hline $\mathrm{P} 6$ & $\mathrm{CH} 03 \mathrm{~d} 12-\mathrm{F}$ & GCCCAGAAGCAATAAGTAAACC & $\mathrm{CH} 03 \mathrm{~d} 12-\mathrm{R}$ & ATTGCTCCATGCATAAAGGG \\
\hline P7 & $\mathrm{CH} 04 \mathrm{e} 05-\mathrm{F}$ & AGGCTAACAGAAATGTGGTTTG & $\mathrm{CH} 04 \mathrm{e} 05-\mathrm{R}$ & ATGGCTCCTATTGCCATCAT \\
\hline P8 & CH01hl0-F & TGCAAAGATAGGTAGATATATGCCA & CH01h10-R & AGGAGGGATTGTTTGTGCAC \\
\hline P9 & $\mathrm{CH} 05 \mathrm{c} 07-\mathrm{F}$ & TGATGCATTAGGGCTTGTACTT & $\mathrm{CH} 05 \mathrm{c} 07-\mathrm{R}$ & GGGATGCATTGCTAAATAGGAT \\
\hline P10 & $\mathrm{NH} 017 \mathrm{a}-\mathrm{F}$ & CAGAAAGGAGAGGGCTACAG & NH017a-R & СССТСАСССААТСААААСТС \\
\hline P11 & $\mathrm{CH} 03 \mathrm{~d} 02-\mathrm{F}$ & AАACTTTCACTTTCACCCACG & $\mathrm{CH} 03 \mathrm{~d} 02-\mathrm{R}$ & ACTACATTTTTAGATTTGTGCGTC \\
\hline P12 & $\mathrm{CH} 01 \mathrm{f0} 2-\mathrm{F}$ & ACCACATTAGAGCAGTTGAGG & $\mathrm{CH} 01 \mathrm{f02}-\mathrm{R}$ & CTGGTTTGTTTTCСTCCAGC \\
\hline P13 & NH009b-F & CCGAGCACTACCATTGA & NH009b-R & CGTCTGTTTACCGCTTCT \\
\hline P14 & NH004a-F & AGGATGGGACGAGTTTAGAG & NH004a-R & ССАСАТСТСТСААССТАССА \\
\hline P15 & $\mathrm{CH} 02 \mathrm{~d} 11-\mathrm{F}$ & AGCGTCCAGAGCAACAGC & $\mathrm{CH} 02 \mathrm{~d} 11-\mathrm{R}$ & AACAAAAGCAGATCCGTTGC \\
\hline P16 & NH007b-F & TACCTTGATGGGAACTGAAC & NH007b-R & ATAGTAGATTGCAATTACTC \\
\hline P17 & NH015a-F & TTGTGCCCTTTTTCCTACC & NH015a-R & CTTTGATGTTACCCCTTGCTG \\
\hline
\end{tabular}


Table 3. Sequences of oligonucleotide adapters and primer combinations used in AFLP

\begin{tabular}{|c|c|c|c|c|}
\hline No. & $\begin{array}{l}\text { Primer combinations and } \\
\text { adapters }\end{array}$ & $\begin{array}{c}\text { Sequences } \\
\left(5^{\prime}-3^{\prime}\right)\end{array}$ & $\begin{array}{l}\text { Primer combinations and } \\
\text { adapters }\end{array}$ & $\begin{array}{c}\text { Sequences } \\
\left(5^{\prime}-3^{\prime}\right)\end{array}$ \\
\hline & E00 & GACTGCGTACCAATTC & M00 & GATGAGTCCTGAGTAA \\
\hline & EcoR I adapter 1 & CTCGTAGACTGCGTACC & Eco R I adapter2 & AATTGGTACGCAGTCTAC \\
\hline & Mse I adapter 1 & GACGATGAGTCCTGAG & Mse I adapter2 & TACTCAGGACTCAT \\
\hline & EcoR I primer & & Mse I primer & \\
\hline $\mathrm{Al}$ & E75 & GACTGCGTACCAATTCGTA & M62 & GATGAGTCCTGAGTAACTT \\
\hline $\mathrm{A} 2$ & E83 & GACTGCGTACCAATTCTCG & M50 & GATGAGTCCTGAGCGGCAT \\
\hline A3 & E32 & GACTGCGTACCAATTCAAC & M66 & GATGAGTCCTGAGTAAGAT \\
\hline A4 & E77 & GACTGCGTACCAATTCGTG & M49 & GATGAGTCCTGAGTAACAG \\
\hline A5 & E84 & GATGAGTCCTGAGTAATCC & M62 & GATGAGTCCTGAGTAACAG \\
\hline A 6 & E85 & GACTGCGTACCAATTCGTG & M50 & GATGAGTCCTGAGCGGCAT \\
\hline A7 & E37 & GACTGCGTACCAATTCACG & M66 & GATGAGTCCTGAGTAAGAT \\
\hline A8 & E59 & GACTGCGTACCAATTCCTA & M60 & GATGAGTCCTGAGCGGCTC \\
\hline A9 & E86 & GATGAGTCCTGAGTAATCT & M49 & GATGAGTCCTGAGTAACAG \\
\hline Al0 & E42 & GACTGCGTACCAATTCCTA & M51 & GATGAGTCCTGAGCGGCAT \\
\hline
\end{tabular}

\section{Data analysis}

Data was analyzed using GeneMarker 2.2 software to display the fragment sizes as electropherograms and binary data. The NTSYSpc 2.11 software package was used to calculate the individual similarity coefficient (ISC) and cluster analysis was performed by UPGMA method. The genetic diversity of the samples was analyzed by Popgene and NTSYS software. Structure 2.3.4 software was used to analyze the data of SSR and AFLP to obtain the group structure diagram of SSR and AFLP (Evanno et al., 2005; Falush et al., 2007).

\section{Results}

\section{Amplification of SSRs and AFLPs}

For SSR analysis, 17 pairs of SSR primers were tested in four $P$. pyrifolia 'Nakai' varieties ('Eli 2', 'Housui', 'Jingli 1' and 'Wonhwang') in order to identify candidate primers. The results showed that polymorphism of clear bands was generated among the total analyzed sample (Fig. 1). Finally, 10 pairs of SSR polymorphic primers were used to amplify $P$. pyrifolia 'Nakai' samples, and a total of 90 alleles were detected (Table 4). The number of alleles detected by each pair of primers was between 5.0 and 12.0, with an average of 9.0 (Table 4). The actual number of alleles detected by each primer was 5.0686. The polymorphism information content indexes (PIC) were between 0.5578 and 0.8423 , with an average of 0.7585 , indicating that the polymorphism information content indexes of the primers were quite different.

The 10 pairs of the selected AFLP combined primers were used for PCR amplification of the P. pyrifolia 'Nakai' DNA samples. As shown in Table 5, the average amplification results of each primer combination could detect 105 bands, 169 bands at most. In the currently reported AFLP technology based on silver staining, the number of bands amplified by most primer combinations was approximately 50 , and the maximum number was not more than 100. Our results showed that the number of bands detected by AFLP in this experiment was significantly more than that reported in the literatures (Bao et al., 2008; Monte-Corvo et al., 2000), which confirmed the abundance of genetic diversity of pear varieties and enhances the reliability of this technique in analyzing such diversity. Moreover, the detection rate of polymorphism was also quite high. The polymorphic bands of 10 pairs of primer combinations among $P$. pyrifolia 'Nakai' varieties were 1,046 with an average polymorphic percentage of $86.46 \%$. The primer combination with the highest polymorphism is E84M62, with a polymorphism percentage of $92.24 \%$. Therefore, specific genetic differences exist among $P$. pyrifolia 'Nakai' varieties.

\section{Cluster analysis}

The results of SSR analysis showed that the genetic distance or genetic similarity coefficient between these four varieties 'Deshengxiang', 'Eli 1', 'Yuxiang', and 'Zhongxiang') and other varieties is 0 . Thus, the results of SSR cluster and population structure maps do not include these four varieties. As shown in Fig. 2, when the genetic similarity coefficient was 0.30 , the results of SSR dendrogram analysis showed that 26 varieties can be divided into cluster I (SSR-I), cluster II (SSR-II), and cluster III (SSR-III). SSR-I covered 19 materials, accounting for $73 \%$ of the total materials, including 12 domestic varieties ('Chuxiang', 'Yulv', 'Huali 1', 'Xueqiu', 'Jinshui 3', 'Cuiguan', 'Jinshui 2', 'Eli 2', 'Jingli 1', 'Xizilv', 'Qingxiang', and 'Huangguan') and 7 foreign varieties ('Ninomiyahuri', 'Qiuyue', 'Housui', 'Wonhwang,' 'Whangkeumbae', 'Chojuro', and 'Niitaka'). SSR-II contained 3 domestic varieties ('Jinshuiqiu', 'Laiyangcili', and 'Jinshuisu'), accounting for $12 \%$ of the total varieties. SSR-III covered 4 domestic varieties ('Lvbaoshi', 'Zaosu', 'Zaomeisu', and 'Wuzili'), accounting for $15 \%$ of the total varieties.

According to the data of genetic similarity coefficient, when the population similarity coefficient exceeded 0.72 , the genetic differentiation between different varieties becomes larger and larger, and was divided into three clusters (Fig. 3). P. pyrifolia 'Nakai' varieties can be divided into cluster I (AFLP-I), cluster II (AFLP-II), and cluster III (AFLP-III). AFLP-I consisted of 11 varieties ('Chuxiang', 'Whangkeumbae', 'Jingli 1', 'Cuiguan', 'Niitaka', 'Chojuro', 'Jinshui 3', 'Huali 1', 'Yuxiang', 'Laiyangcil'I, and 'Ninomiyahuri'), accounting for $37 \%$ of the total varieties. This cluster included most Chinese varieties and a few foreign varieties. AFLP-II consisted of 13 varieties ('Deshengxiang', 'Yulv', 'Jinshui 2', 'Eli 1', 'Eli 2', 'Housui', 'Wonhwang', 'Huangguan', 'Jinshuiqiu', 'Zaosu', 
974

$\begin{array}{llllllllllllllllll}\text { P1 } & \text { P2 } & \text { P3 } & \text { P4 } & \text { P5 } & \text { P6 } & \text { P7 } & \text { P9 } & \text { P10 } & \text { P11 } & \text { P13 } & \text { P16 }\end{array}$

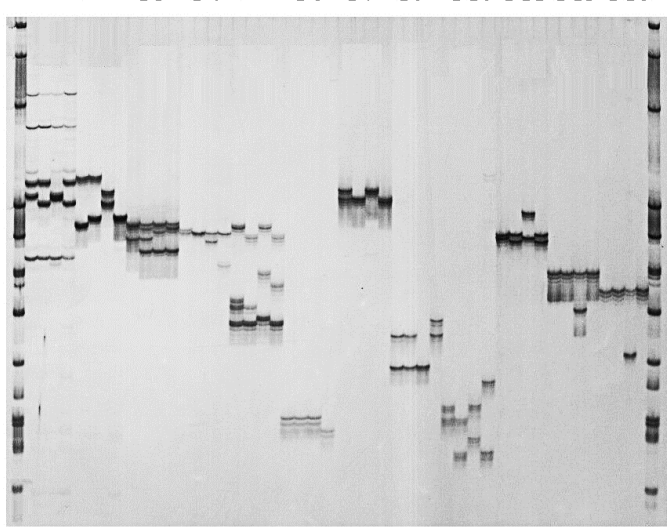

P8 $\quad$ P12 P14 P15 P17

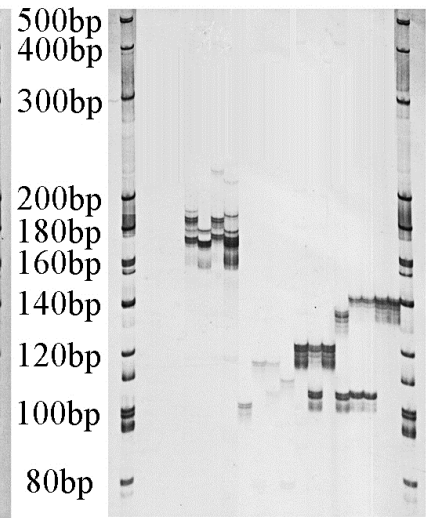

Fig. 1. Four $P$. pyrifolia 'Nakai' varieties ('Eli 2', 'Housui', 'Jingli 1' and 'Wonhwang') were amplified by 17 SSR primer pairs

Table 4. Analysis of polymorphic bands of 10 pairs of SSR primers

\begin{tabular}{ccccccccc}
\hline Code & Locus name & $\mathrm{Na}$ & $\mathrm{Ne}$ & $\mathrm{I}$ & $\mathrm{Ho}$ & $\mathrm{He}$ & Fst & PIC \\
\hline P1 & NH013a & 9 & 5.4516 & 1.8892 & 0.8462 & 0.8326 & 0.4819 & 0.7931 \\
P2 & BGT23b & 9 & 4.3613 & 1.7680 & 0.7692 & 0.7858 & 0.5010 & 0.7460 \\
P4 & NH011a & 10 & 4.8828 & 1.8946 & 0.8000 & 0.8114 & 0.5255 & 0.7750 \\
P7 & CH04405 & 11 & 6.5631 & 2.0787 & 0.9231 & 0.8643 & 0.4555 & 0.8302 \\
P9 & CH05c07 & 8 & 3.5767 & 1.5286 & 0.6538 & 0.7345 & 0.5462 & 0.6781 \\
P10 & NH017a & 8 & 5.8276 & 1.8836 & 0.7692 & 0.8446 & 0.5357 & 0.8067 \\
P11 & CH03d02 & 12 & 6.9691 & 2.1755 & 0.9231 & 0.8733 & 0.4611 & 0.8423 \\
P14 & NH004a & 10 & 6.2304 & 1.9867 & 0.8462 & 0.8560 & 0.4960 & 0.8198 \\
P16 & NH007b & 5 & 2.5177 & 1.1629 & 0.6154 & 0.6146 & 0.4896 & 0.5578 \\
P17 & NH015a & 8 & 4.3057 & 1.6909 & 0.7308 & 0.7828 & 0.5241 & 0.7362 \\
Mean & & 9 & 5.0686 & 1.8059 & 0.7877 & 0.8000 & 0.5009 & 0.7585 \\
\hline Note: Na-Observed number of alleles: Ne-Effective number of alleles; I-Shannon's Information: Ho-Observedheterozygosity: He-Expectedheterozygosity: Fst-Genetic
\end{tabular}

Note: Na-Observed number of alleles; Ne-Effective number of alleles; I-Shannon's Information; Ho-Observed heterozygosity; He-Expected heterozygosity; Fst-Genetic differentiation coefficient; PIC-The percentage of polymorphic loci.

Table 5. Analysis of polymorphic bands of 10 pairs of AFLP primers

\begin{tabular}{cccccccc}
\hline No. & Primer combination & $\mathrm{Na}$ & $\mathrm{Ne}$ & $\mathrm{Ho}$ & $\mathrm{I}$ & $\mathrm{P} 1$ & $\mathrm{P} 2(\%)$ \\
\hline A1 & E75M62 & 1.9118 & 1.5569 & 0.3232 & 0.4820 & 93 & 91.18 \\
A2 & E83M50 & 1.5854 & 1.3586 & 0.2087 & 0.3108 & 72 & 58.54 \\
A3 & E32M66 & 1.8759 & 1.5712 & 0.3283 & 0.4859 & 120 & 87.59 \\
A4 & E77M49 & 1.9217 & 1.5546 & 0.3253 & 0.4869 & 106 & 92.17 \\
A5 & E84M62 & 1.8895 & 1.5212 & 0.3151 & 0.4753 & 169 & 88.95 \\
A6 & E85M50 & 1.9224 & 1.6145 & 0.3556 & 0.5248 & 107 & 92.24 \\
A7 & E37M66 & 1.8939 & 1.6138 & 0.3516 & 0.5167 & 118 & 89.39 \\
A8 & E59M60 & 1.8796 & 1.5826 & 0.3388 & 0.5007 & 95 & 87.96 \\
A9 & E86M49 & 1.8842 & 1.6002 & 0.3426 & 0.5039 & 84 & 88.42 \\
A10 & E42M51 & 1.8817 & 1.6123 & 0.3486 & 0.5112 & 82 & 88.17 \\
Mean & & 1.8646 & 1.55859 & 0.3238 & 0.4798 & 104.6 & 86.46 \\
\hline
\end{tabular}

Note: P1-Polymorphic bands, P2-Percentage of polymorphic bands

'Zaomeisu', 'Jinshuisu', and 'Zhongxiang'), accounting for $43 \%$ of the total varieties. AFLP-III covers 6 varieties' ('Lvbaoshi', 'Qingxiang', 'Qiuyue', 'Wuzili', 'Xueqiu', and 'Xizilv'), accounting for $20 \%$ of the total varieties.

SSR and AFLP data were combined for UPGMA clustering analysis. The genetic similarity coefficient were 0.72 , which was expressed in cluster I (SSR+AFLP)-I, cluster II (SSR+AFLP)-II, and cluster III (SSR+AFLP)-III (Fig. 4). The first major cluster (SSR+AFLP)-I included 6 varieties, accounting for $20 \%$ of the total varieties, which included 5 domestic varieties ('Chuxiang', 'Zaosu', 'Huali 1', 'Jingli 1', and 'Jinshui 3') and 1 Korean variety
('Whangkeumbae'). The second cluster (SSR+AFLP)-II contained 18 varieties, accounting for $60 \%$ of the total varieties, including 13 domestic varieties ('Cuiguan', 'Zaomeisu', 'Laiyangcili', 'Huangguan', 'Jinshuiqiu', 'Zhongxiang', 'Jinshuisu', 'Yuxiang', 'Jinshui 2', 'Deshengxiang', 'Yulv', 'Eli 1', and 'Eli 2') and 5 imported varieties 'Niitaka', 'Chojuro', 'Ninomiyahuri', 'Wonhwang', and 'Housui'). The third cluster (SSR+AFLP)-III contained 6 varieties, accounting for $20 \%$ of the total varieties, covering 5 Chinese varieties ('Lvbaoshi', 'Qingxiang', 'Wuzili', 'Xueqiu', and 'Xizilv') and 1 Japanese variety ('Qiuyue'). 


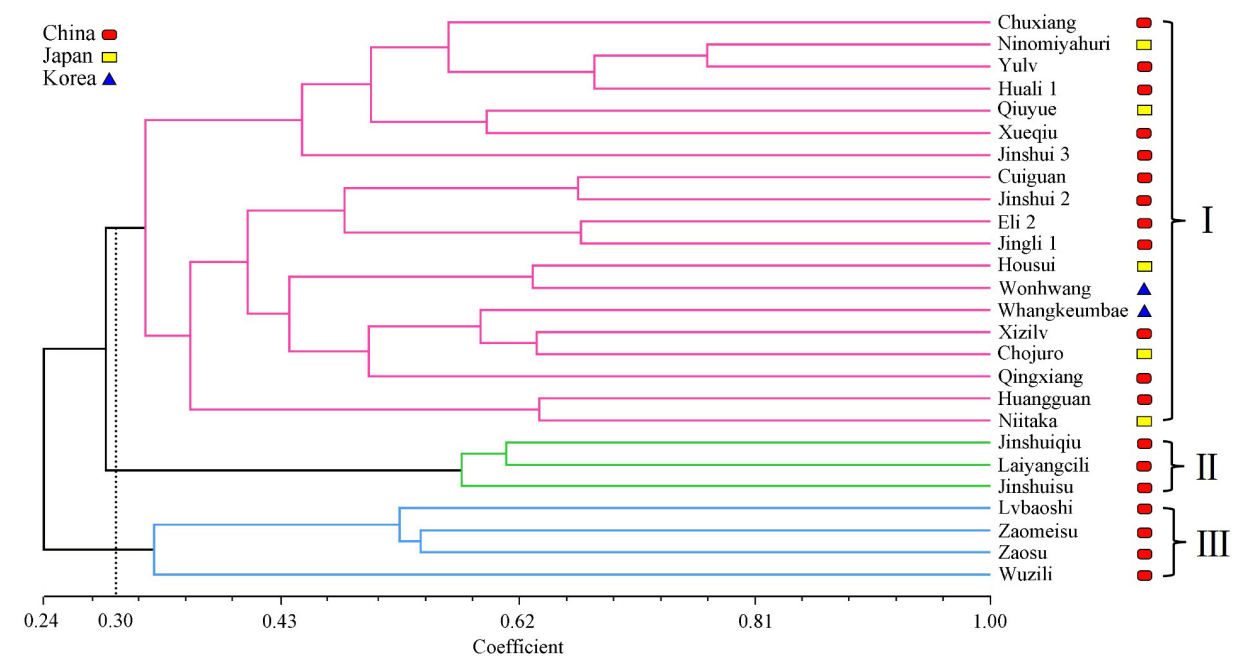

Fig. 2. Dendogram obtained by cluster analysis based on SSR data

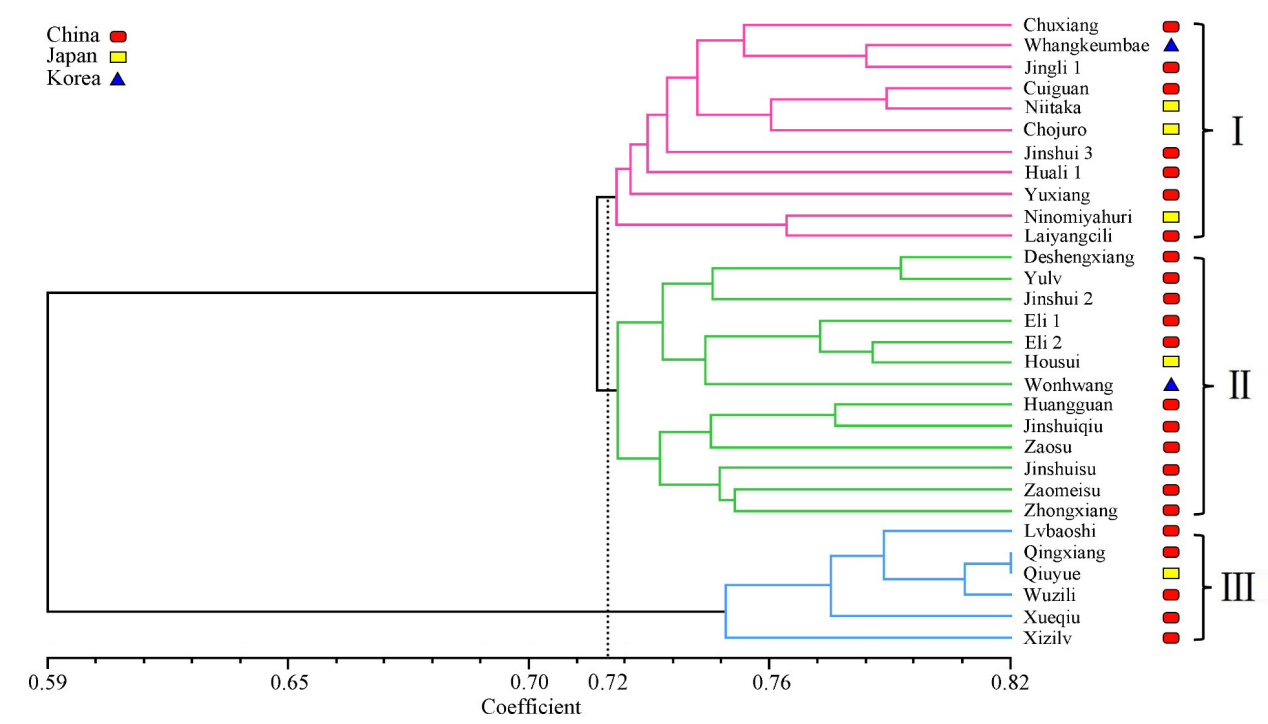

Fig. 3. Dendogram obtained by cluster analysis based on AFLP data

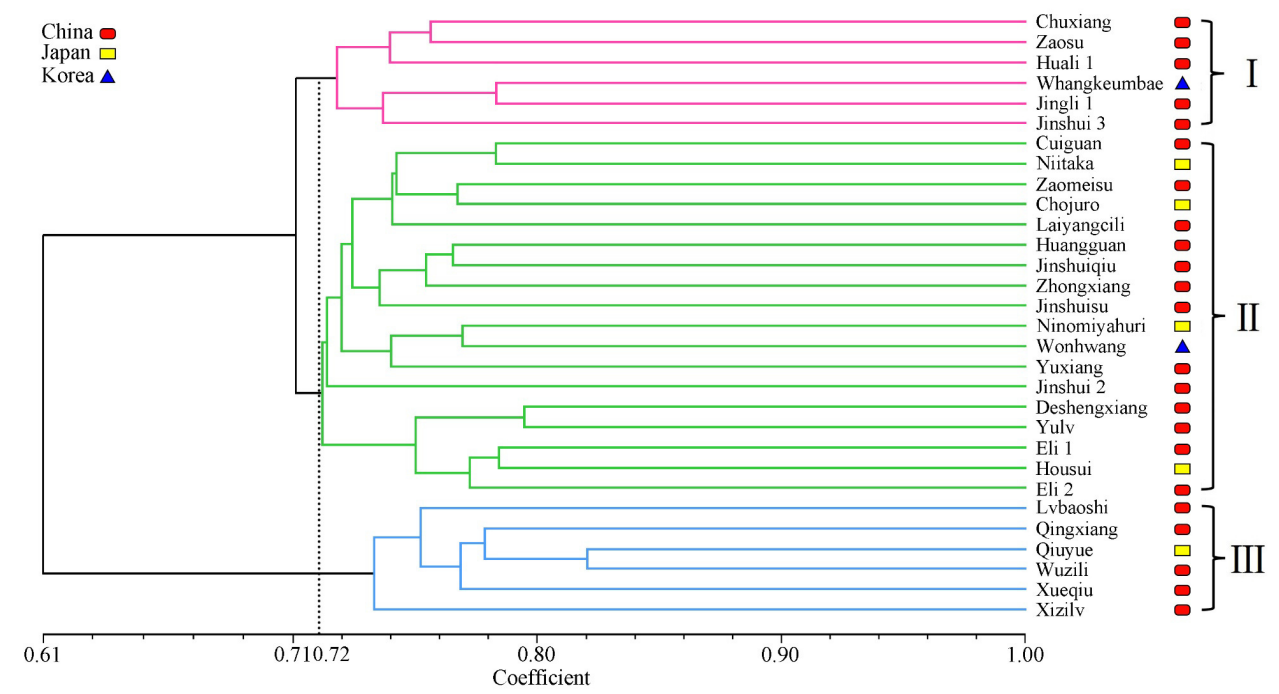

Fig. 4. Dendogram obtained by cluster analysis based on SSR and AFLP data 
976

\section{Genetic structure}

Structure 2.3.4 software was used to analyze the data of SSR and AFLP, and the optimal $\mathrm{K}$ values of both SSR and AFLP were 2 (Fig. 5). In other words, both SSR and AFLP populations contained two genotypes, which were expressed by PK and PA. Each of the two genotypes in the SSR population structure diagramed account for half (Fig. 6), which was consistent with Fst in SSR genetic variation analysis (Table 4). Both were heterozygotes in the population, and no pure breed exists. SSR-I contained two genotypes, PK and PA, of which the proportion of the PK genotype was slightly larger than that of the PA genotype. However, the proportion of PA in P. pyrifolia 'Nakai' varieties in SSR-II and SSR-III was much larger than that in PK.

The results of population structure analysis showed that the PK genotype was introgression in the two pear varieties because a small amount of PK gene was doped in ancestral parents of the two pears during the breeding process. The

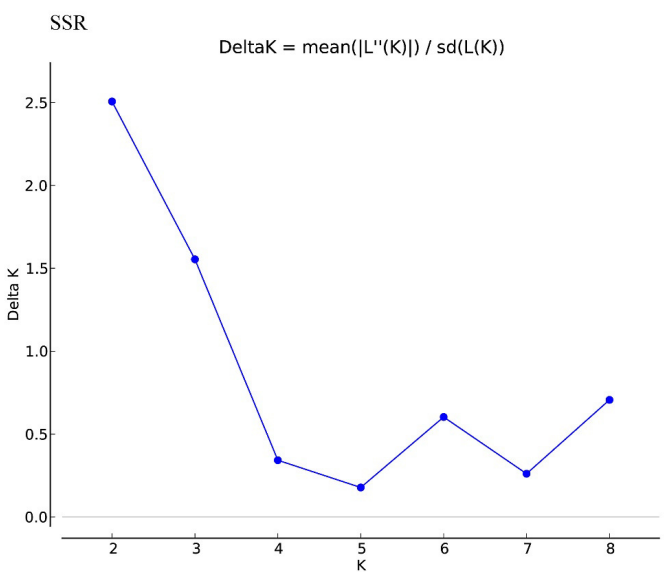

SSR population structure map showed the rich background of genetic diversity of $P$. pyrifolia 'Nakai' varieties we have studied, thereby further verifying the PIC value of SSR polymorphism band analysis.

The population structure results analyzed by AFLP were presented in Fig. 7. The proportion of PK in the population was much larger than PA, which was consistent with the gene diversity index Ho in AFLP polymorphism analysis (Table 5). Overall, the $P$. pyrifolia 'Nakai'varieties included 18 purebreds, of which 14 varieties ('Cuiguan', 'Whangkeumbae', 'Jinshui 3', 'Laiyangcili', 'Chuxiang', 'Deshengxiang', 'Housui', 'Huangguan', 'Jinshui 2', 'Jinshuiqiu', 'Qingxiang', 'Xizilv', 'Qiuyue', and 'Xueqiu') only contained PK. According to the genetic background, almost all parents were Japanese pears or local varieties. The genotype of the other four varieties ('Yuxiang', 'Wonhwang', 'Zaomeisu', and 'Zaosu') was PA. Except 'Yuxiang', the three other varieties were clustered on AFLPII, which further verified the clustering accuracy of AFLP.

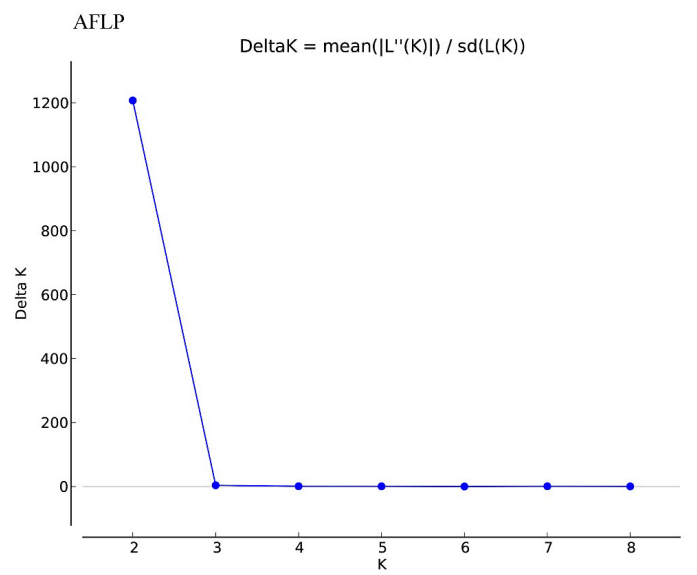

Fig. 5. Estimation of the most probable K value for the P. pyrifolia 'Nakai' cultivars, based on the method of Evano (2005)

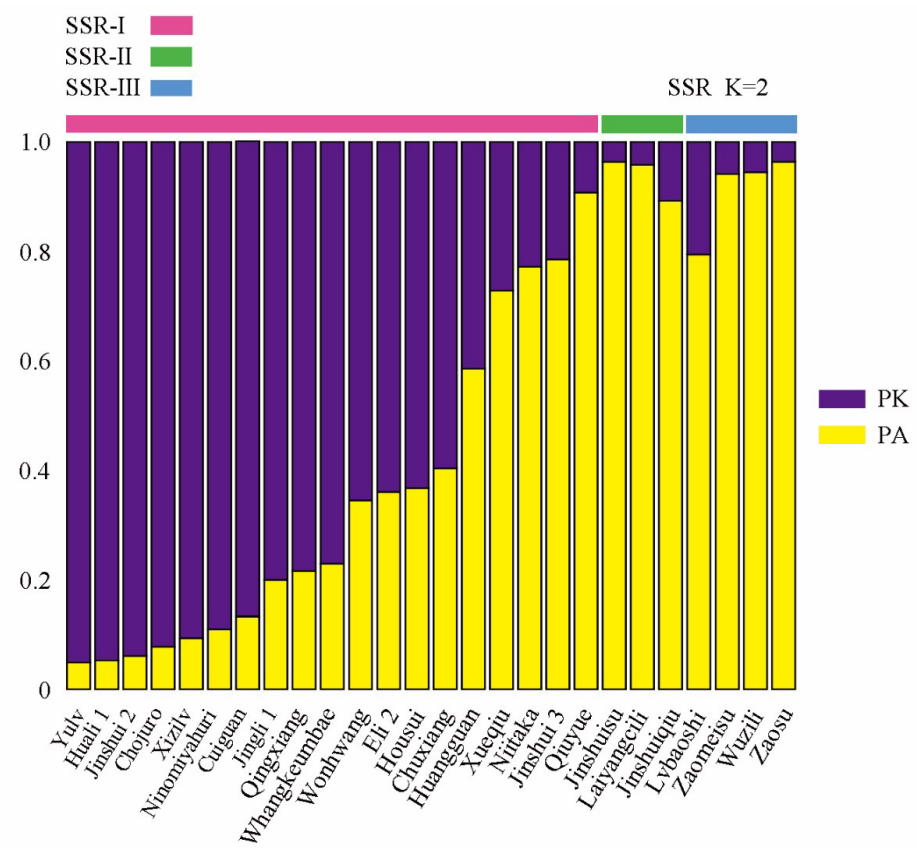

Fig. 6. Genotype class assignment of 26 Pyrus pyrifolia 'Nakai' varieties based on the software Structure2.3.4 using SSR 
AFLP-I

AFLP-II

AFLP-III

AFLP $\quad K=2$

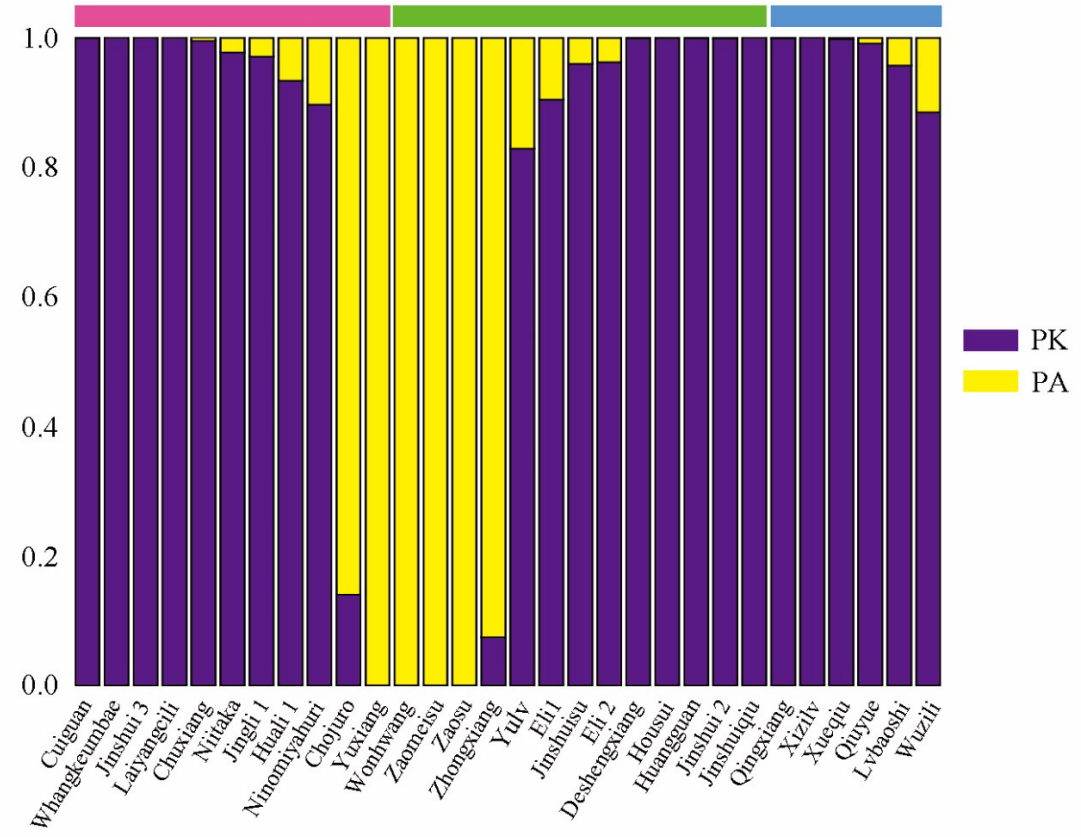

Fig. 7. Genotype class assignment of all Pyrus pyrifolia 'Nakai' varieties based on the software Structure 2.3.4 using SSR

\section{Discussion}

SSR and AFLP have been used in variety identification and genetic diversity determination of various fruit trees. Therefore, this study combined SSR and AFLP marker technology to analyze the genetic diversity among pear varieties. Here, SSR (75.85\%) and AFLP (86.46\%) in selected $P$. pyrifolia 'Nakai' varieties have shown high polymorphism similar to that of the markers in other fruit tree studies: $75.24 \%$ in 8 mango genotypes (384 SSRs) (Song et al., 2015), 71.00\% in 9 coconut genotypes (171 SSRs) (Loiola et al., 2016), 76.30\% in 27 papaya genotypes (378 SSRs) (Sengupta et al., 2014), 87\% in 27 plum accessions (134 SSRs) (Pop et al., 2018), 73.50\% in 162 pineapple genotypes (377 AFLPs) (Kato et al., 2005), $78.34 \%$ in 137 Adansonia digitata 'Linn'. genotypes (217 AFLPs) (Assogbadjo et al., 2006), and $53.00 \%$ in 16 banana genotypes (148 AFLPs) (Loh et al., 2000). The above results showed that both SSR and AFLP were ideal molecular markers, which could effectively evaluate the genetic diversity of $P$. pyrifolia 'Nakai' varieties.

Although the clustering results of $P$. pyrifolia 'Nakai' varieties in different marker results were not completely consistent, the three tree clustering maps generated from SSR, AFLP, and combination data showed approximate similarity. Most of the varieties in (SSR+AFLP)-II correspond to those in SSR-II and AFLP-II, included 8-9 identical domestic varieties. Furthermore, (SSR+SRAP)-III germplasm was highly similar to that in the SSR-III germplasm. SSR-I included cluster I of AFLP or SSR+AFLP dendrogram. Notably, the four varieties ('Chuxiang', 'Eli 1', 'Eli 2', and 'Jingli 1') bred by our research group were in the same cluster as most commercial varieties abroad. However, we also found that some varieties were located in different clusters in the three genetic clustering maps; for example, the domestic variety 'Zaosu' was in SSR-I but in AFLP-III and (SSR+SRAP)-II in the other two genetic clustering maps. On the contrary, some varieties were stable in the same cluster in the three genetic clustering maps. For example, 4 domestic varieties ('Chuxiang', 'Huali 1', 'Jingli 1', and 'Jingshui 3') and Korean variety ('Whangkeumbae') were all located in the first cluster in the three genetic clustering maps, and 'Chuxiang' was ranked first in the first cluster. The second cluster of the three genetic clustering maps covered the domestic varieties of 'Jinshuisu' and 'Jinshuiqiu'. However, the domestic varieties of 'Lvbaoshi' and 'Wuzili' were in the third cluster of three genetic clustering maps, and 'Lvbaoshi' always ranked in the first cluster of the third cluster. By observing the genetic background of P. pyrifolia 'Nakai' varieties, we found that 'Nijisseik', 'Ejima, and 'Fuli' were good parent resources. A large amount of $P$. pyrifolia 'Nakai' varieties were bred with these 3 varieties as parents. This discovery can provide theoretical guidance for pear variety breeding and genetic improvement.

In other horticultural plants, genetic diversity analysis was often conducted by combining various types of molecular markers. For example, SSR markers were used to study genetic diversity in tung tree (Zhang et al., 2014), AFLP markers were used to study genetic diversity in pomegranate (Nemati et al., 2012), SSR and AFLP markers were used to study genetic diversity in watermelon (Hwang et al., 2011), SSR and SRAP markers were used to study genetic diversity in lemon (Uzun et al., 2011), SSR markers were used to study genetic diversity in wild apricot (He et 
978

al., 2007). Although the dendrogram generated by using various molecular markers showed high similarity, some differences exist in cluster analysis obtained from various types of markers. The reason may be the use of different marker systems, different marker quantities or different test groups. Different molecular markers have been developed using different principles. Thus, the expected amplified fragments had different locations in the entire genome and the amount of genome coverage. In the same species, sampling variation may vary depending on the quantities. Therefore, the dendrogram constructed on the basis of the molecular markers with more types and quantities is realistic. Therefore, the results obtained by SSR+AFLP dendrogram can more significantly reflect the genetic diversity among $P$. pyrifolia 'Nakai' varieties in this study.

To a certain extent, most ecological plants were clustered according to their geographical distribution. In this experiment, based on cluster analysis of SSR+AFLP data, $70 \%$ of pear varieties from Korea and Japan were combined in cluster II and the remaining 30\% were in the other two clusters. The results of this study showed that all Japanese and Korean varieties were not located in a cluster, and no obvious relationship exists between geographical origin and molecular marker clusters, which was consistent with the findings of Johnson et al. (2002). The genetic structure diagram showed that $P$. pyrifolia 'Nakai' varieties contained two genotypes, because no significant correlation existed between the geographical origin of varieties and its molecular marker clusters (Yuan et al., 2018). The reason may be the diffusion of introduced species, hybridization through geographically different genetic populations as parents, and potential inherent genetic overlaps that may exist in these germplasms (An et al., 2017).

\section{Conclusions}

It was concluded that the $P$. pyrifolia 'Nakai' varieties shared high genetic similarity and abundant genetic diversity. In addition, the genetic background analysis of $P$. pyrifolia 'Nakai' varieties suggested that 'Nijisseik', 'Ejima', and 'Fuli' were good parent resources among P. Pyrifolia 'Nakai' varieties varieties. Due to the communication between varieties of $P$. pyrifolia 'Nakai' in different regions, the genetic diversity of $P$. pyrifolia 'Nakai' varieties increases, which leads to the increase of genetic diversity among varieties of $P$. pyrifolia 'Nakai'. Therefore, these results can provide useful references for further research on molecular identification and conservation of germplasm resources, as well as pear variety breeding and genetic improvement.

\section{Acknowledgements}

This work was supported by The Special Projects for Technological Innovation in Hubei Province (Grant No. 2019ABA113).

\section{Conflicts of Interest}

The authors declare that there are no conflicts of interest related to this article.

\section{References}

An M, Deng M, Zheng SS, Jiang XI, Song YG (2017). Introgression threatens the genetic diversity of Quercus austrocochinchinensis (Fagaceae), an endangered oak: a case inferred by molecular markers. Frontiers in Plant Science 8:229.

Assogbadjo AE, Kyndt T, Sinsin B, Gheysen G, Damme PV (2006). Patterns of genetic and morphometric diversity in baobab (Adansonia digitata) populations across different climatic zones of Benin (West Africa). Annals of Botany 97(5):819-830.

Bao L, Chen K, Zhang D, Li X, Teng Y (2008). An assessment of genetic variability and relationships within Asian pears based on AFLP (amplified fragment length polymorphism) markers. Scientia Horticulturae 116(4):374380.

Celton JM, Tustin DS, Chagné D, Gardiner SE (2009). Construction of a dense genetic linkage map for apple rootstocks using SSRs developed from Malus ESTs and Pyrus genomic sequences. Tree Genetics and Genomes 5(1):93-107.

ChengZ, Huang H (2009). SSR fingerprinting Chinese peach cultivars and landraces (Prunus persica) and analysis of their genetic relationships. ScientiaHorticulturae 120(2):188-193.

Evanno G, Regnaut S, Goudet J (2005). Detecting the number of clusters of individuals using the software structure: a simulation study. Molecular Ecology 14(8):2611-2620.

Falush D, Stephens M, Pritchard JK (2007). Inference of population structure using multilocus genotype data: dominant markers and null alleles. Molecular Ecology Notes 7(4):574-578.

Güney M, Kafkas S, Keles H, Aras S, Ercişli S (2018). Characterization of hawthorn (Crataegus spp.) genotypes by SSR markers. Physiology and Molecular Biology of Plants 24(6): 1221-1230.

He TM, Chen XS, Xu Z, Gao JS, Lin PJ, Liu W, ... Wu Y (2007). Using SSR markers to determine the population genetic structure of wild apricot (Prunus armeniaca L.) in the Ily Valley of West China. Genetic Resources and Crop Evolution 54(3):563-572.

Hwang J, Kang J, Son B, Kim K, Park Y (2011). Genetic diversity in watermelon cultivars and related species based on AFLPs and ESTSSRs. Notulae Botanicae Horti Agrobotanici Cluj-Napoca 39(2):285292.

Jinbo U, Kato T, Ito M (2011). Current progress in DNA barcoding and future implications for entomology. Entomological Science 14(2):107124.

Johnson RC, Johnston WJ, Golob CT, Nelson MC, Soreng RJ (2002). Characterization of the USDA Poa pratensis collection using RAPD markers and agronomic descriptors. Genetic Resources and Crop Evolution 49(4):351-363.

Kato CY, Nagai C, Moore PH, Zee F, Kim MS, Steiger DL, ... Ming R (2005). Intra-specific DNA polymorphism in pineapple (Ananas comosus (L.) Merr.) assessed by AFLP markers. Genetic Resources and CropEvolution 51(8):815-825.

Liebhard R, Gianfranceschi L, Koller B, Ryder CD, Tarchini R, WegEVD, Gessler C (2002). Development and characterisation of 140 new microsatellites in apple (Malus $\times$ domestica Borkh.). Molecular Breeding $10(4): 217-241$. 
Lisek A, Rozpara E (2010). Identification of pear cultivars with RAPD and ISSR markers. Journal of Fruit and Ornamental Plant Research 18(2):17-22.

Loh JP, Kiew R, Set O, Gan LH, Gan YY (2000). Amplified fragment length polymorphism fingerprinting of 16 banana cultivars (Musa cvs.). Molecular Phylogenetics and Evolution 17(3):360-366.

Loiola CM, Azevedo AON, Diniz LEC, Aragão WM, Azevedo CDDO, Santos PHAD, ... Ramos SRR (2016). Genetic relationships among tall coconut palm (Cocos nuifera L.) accessions of the International Coconut Genebank for Latin America and the Caribbean (ICGLAC), evaluated using microsatellite markers (SSRs). PLoS One 11(3):191-201.

Monte-Corvo L, Goulão L, Oliveira C (2001). ISSR analysis of cultivars of pear and suitability of molecular markers for clone discrimination. Journal of the American Society for Horticultural Science 126(5):517522.

Monte-Corvo L, Cabrita L, Oliveira C, Leitão J (2000). Assessment of genetic relationships among Pyrus species and cultivars using AFLP and RAPD markers. Genetic Resources and Crop Evolution 47(3):257265.

Nemati Z, Tehranifar A, Farsi M, Kakhki AM, Nemati H, Khayat M (2012). Evaluation of genetic diversity of Iranian pomegranate cultivars using fruit morphological characteristics and AFLP markers. Notulae Botanicae Horti Agrobotanici Cluj-Napoca 40(1):261-268.

Poczai P, Varga I, Laos M, Cseh A, Bell N, Valkonen JP, Hyvönen J (2013). Advances in plant gene-targeted and functional markers: a review. Plant Methods 9(1):6.

Pop R, Harta M, Szabo K, Zânescu M, Sisea CR, Catana C, Pamfil D (2018). Genetic diversity and population structure of plum accessions from a Romanian germplasm collection assessed by simple sequence repeat (SSR) markers. Notulae Botanicae Horti Agrobotanici ClujNapoca 46(1):90-96.

Puskás M,Höfer M, Sestras RE, Peil A, Sestras AF, Hanke M-V, Flachowsky $\mathrm{H}$ (2016). Molecular and flow cytometric evaluation of pear (Pyrus L.) genetic resources of the German and Romanian national fruit collections. Genetic Resources and CropEvolution 63(6):1023-1033.

Rao MN, Soneji JR, Chen C, Huang S, Gmitter FG (2008). Characterization of zygotic and nucellar seedlings from sour orange-like citrus rootstock candidates using RAPD and EST-SSR markers. Tree GeneticsandGenomes 4(1):113-124.

Sánchez-Sevilla JF, Horvath A, Botella MA, Gaston A, Folta K, Kilian A, ... Amaya I (2015). Diversity Arrays Technology (DArT) marker platforms for diversity analysis and linkage mapping in a complex crop, the octoploid cultivated strawberry (Fragaria $\times$ ananassa). PLoS One 10(12):e0144960.

Sengupta S, Das B, Acharyya P, Prasad M, Ghose TK (2014). Genetic diversity analysis in a set of caricaceae accessions using resistance gene analogues. BMC Genetics 15(1):137.

SongX, Ge T, LiY, Hou X(2015). Genome-wide identification ofSSR and SNP markers from the non-heading Chinese cabbage for comparative genomic analyses. BMC Genomics 16(1):328.
Tana W, Hitomi A, Xu J, Teng M, Chiyomi U, Hironori K (2015). Population structure of and conservation strategies for wild Pyrus Ussuriensis Maxim. in China. PLoSOne 10(8):e0133686.

Teng Y, Tanabe K (2004). Reconsideration on the origin of cultivated pears native to East Asia. Acta Horticulturae (634):175-182.

TerakamiS, Kimura T,Nishitani C, Sawamura Y,Saito T, Hirabayashi T,... Yamamoto T (2009). Genetic linkage map of the Japanese pear 'Housui' identifying three homozygous genomic regions. Journal of the Japanese Society for Horticultural Science 78(4):417-424.

Uzun A, Yesiloglu T, Polat I, Akakacar Y, Gulsen O, Yildirim B, ... Anil S (2011). Evaluation of genetic diversity in lemons and some of their relatives based on SRAP and SSR markers. Plant Molecular Biology Reporter 29(3):693-701.

Yamamoto T, Kimura T, Shoda M, Imai T, Saito T, Sawamura Y, ... Matsuta N (2002). Genetic linkage maps constructed by using an interspecific cross between Japanese and European pears. Theoretical and Applied Genetics 106(1):9-18.

Yamamoto T, Kimura T, TerakamiS, Nishitani C, Sawamura Y, Saito T,... Hayashi T (2007). Integrated reference genetic linkage maps of pear based on SSR and AFLP markers. Breeding Science 57(4):321-329.

Ye J, Xu F, Wang G, Chen Q (2017). Molecular cloning and characterization of an anthocyanidin synthase gene in Prunus persica (L.) Batsch. Notulae Botanicae Horti Agrobotanici Cluj-Napoca 45(1):2835.

Yuan X, Tu M, He Y, Wang W, Li J, Zhou S (2018). Analysis of Genetic Diversity in 73 Kentucky Bluegrass Materials by SSR and SRAP Markers. Notulae Botanicae Horti Agrobotanici Cluj-Napoca 46(2):327-335.

Yue X, Zheng X, Zong Y, Jiang S, Hu C, Yu P, ... Teng, Y (2018). Combined analyses of chloroplast DNA haplotypes and microsatellite markers reveal new insights into the origin and dissemination route of cultivated pears native to East Asia. Frontiers in Plant Science 9:591.

Yue XY, Liu GQ, Zong Y, Teng YW, Cai DY (2014). Development of genic SSR markers from transcriptome sequencing of pear buds. Journal ofZhejiangUniversity Science B 15(4):303-312.

Zhang L, Jia B, Tan X, Thammina CS, Long H, Liu M, ... Cao H (2014). Fatty acid profile and unigene-derived simple sequence repeat markers in tungtree (Verniciafordii).PLoS One 9(8): e105298.

Zhang MY, Xue C, Xu L, Sun H, Qin MF, ZhangS, WuJ (2016). Distinct transcriptome profiles reveal gene expression patterns during fruit development and maturation in five main cultivated species of pear (PyrusL.).Scientific Reports 6:28130.

Zhang RP, Wu J, Li XG, Khan MA, Chen H, Korban SS, Zhang SL (2012). An AFLP, SRAP, and SSR genetic linkage map and identification of QTLs for fruit traits in pear (Pyrus L.). Plant Molecular Biology Reporter 31(3):678-687. 